\title{
Bacteriology in Diabetic Foot Infections: A Multicentre Cross- sectional Study in Malaysia and Comparison with Southeast Asia Countries
}

Kow Ren $\mathrm{Yi}^{1}$

${ }^{1}$ Department of Orthopaedics, Hospital Tengku Ampuan Afzan

Presenter: Kow Ren Yi

Introduction: Diabetes mellitus is a major non-communicable disease in Malaysia and Southeast Asian Countries. In this study, we aimed to determine the microbiological profile of diabetic foot infection and the antibiotic susceptibility in Malaysia and to compare our findings with that from other Southeast Asia countries. Materials and Methods: A cross-sectional study was conducted in three district hospitals (Bentong, Raub and Kuala Lipis) from the $1^{\text {st }}$ January to the $31^{\text {st }}$ December 2016. Microbiological profiles of intra-operative deep tissue and bone samples from diabetic foot infection were included. Studies reporting the microbiological profiles from Southeast Asia countries were reviewed and the data were extracted for comparison. Results: A high incidence of gram negative pathogens was isolated $(73.4 \%)$. The predominant pathogens isolated included Staphylococcus sp (17.5\%), Klebsiella sp (17\%), Pseudomonas sp (15.4\%) and Proteus $s p(13.8 \%)$. Among those gram negative pathogens tested, a high number of them were resistant to ampicillin and amoxicillin/clavulanic acid. They were sensitive to imipenem (98.9\%), tazosin (97.7\%) and ampicillin-sulbactam (84.8\%). All Southeast Asian countries studied except Singapore have high gram negative to gram positive pathogens ratio. Conclusion: The empirical antibiotics should be tailored to the local bacteriological profiles of diabetic foot infection. 